\title{
Planned Activity Description
}

National Cancer Institute

\section{Source}

National Cancer Institute. Planned Activity Description. NCI Thesaurus. Code C93996.

The textual representation of the planned activity. 